\title{
THE AUTHENTICATION OF VIRGIN COCONUT OIL FROM GRAPE SEED OIL AND SOYBEAN OIL USING FTIR SPECTROSCOPY AND CHEMOMETRICS
}

\author{
ABDUL ROHMAN ${ }^{1,2^{*}}$, YAAKOB BIN CHE MAN ${ }^{3}$, MD. EAKUB ALI ${ }^{4}$ \\ ${ }^{1}$ Faculty of Pharmacy, Universitas Gadjah Mada, Yogyakarta 55281 Indonesia, ${ }^{2}$ Research Center of Halal Products, Universitas Gadjah \\ Mada, Yogyakarta 55281, Indonesia, ${ }^{3}$ Halal Products Research Institute, Universiti Putra Malaysia, 43400 UPM, Serdang, Selangor, \\ Malaysia, ${ }^{4}$ Nanotechnology and Catalysis Research Centre (NanoCat), University of Malaya, Kuala Lumpur 50603, Malaysia \\ Email: abdul_kimfar@ugm.ac.id
}

Received: 29 Dec 2018, Revised and Accepted: 12 Feb 2019

\section{ABSTRACT}

Objective: The objective of this study was to develop Fourier transform infrared (FTIR) spectroscopy in combination with chemometrics of multivariate calibration and discriminant analysis (DA) for the authentication of virgin coconut oil (VCO) from grape seed oil (GSO) and soybean oil (SO).

Methods: FTIR spectra of VCO, GSO, SO and its binary mixture of VCO-SO, and VCO-GSO were scanned at mid-infrared region (4000-650 $\left.\mathrm{cm}^{-1}\right)$ using attenuated total reflectance technique. The wavenumbers were selected based on its capability to provide the best prediction models for quantification and classification of adulterants in VCO assisted by multivariate calibrations and DA, respectively.

Results: The results showed that partial least square (PLS) calibration using absorbance values at combined wavenumbers of 1200-900 and 3027$2985 \mathrm{~cm}^{-1}$ revealed reliable method for quantification of GSO in VCO, as indicated by high value of coefficient of determination $\left(\mathrm{R}^{2}\right)$ and low value of root mean square of calibration (RMSEC) and root mean square error of prediction (RMSEP). PLS using FTIR spectra at the combined wavenumbers of 1200-1000 and 3025-2995 $\mathrm{cm}^{-1}$ was suitable for quantitative analysis of SO in VCO. DAwas also successfully used for classification of VCO and VCO added with adulterants of GSO and SO.

Conclusion: FTIR spectroscopy in combination with chemometrics of multivariate calibration and DA offered effective tools for the authentication of VCO.

Keywords: Virgin coconut oil, Chemometrics, Authentication, FTIR spectroscopy

(C) 2019 The Authors. Published by Innovare Academic Sciences Pvt Ltd. This is an open access article under the CC BY license (http://creativecommons.org/licenses/by/4.0/) DOI: http://dx.doi.org/10.22159/ijap.2019v11i2.31758

\section{INTRODUCTION}

Virgin coconut oil (VCO), a tropical plant oil, is an emerging issue in fats and oil industry, especially in Southeast Asian region like Indonesia, Malaysia, and the Philippines due to its property to have some biological activities including antioxidant, anti-thrombotic, and anti-bacteria against Listeria monocytogenes [1,2]. VCO is rich in medium chain fatty acids (MCFA), especially lauric acid (C12:0), and exhibits good digestibility [3]. Combined with menhaden oil, VCO also exhibited the protective effect through reducing the incidence of the mammary tumor during the animal study [4]. VCO is extracted from the fresh and mature kernel of the coconut meat using either dry or wet methods [5], which retained the active compounds in it. Due to these reasons, VCO is more expensive than other edible oils such as palm oil, grape seed oil, and soybean oil which make VCO is prone to be adulterated with vegetable oils.

The authentication of high-quality edible oils like extra virgin coconut oil and VCO is an interesting study, due to the fact that adulteration practice can come deleterious effects toward human health [6]. The adulteration of VCO with lower-priced oils such as soybean oil is motivated by economic reasons to get economical profit. This adulteration practice is a crucial issue, not only for the consumer but also for producers and regulators in field fats and oils [7]. As a consequence, several physicochemical and molecular biology methods have been continuously developed for identification of adulteration practices of high-value fats and oils. Valid and reliable analytical methods have been reported for the authentication of VCO, namely fast gas chromatography [8], differential scanning calorimetry [9], electronic nose [10], nuclear magnetic resonance phosphorus-31 $\left(31^{\mathrm{P}}\right.$ NMR) spectroscopy [11], and two-dimensional gas chromatography (GC x GC) coupled with time flight-mass spectrometry (TOF-MS) [12] however, these methods required sophisticated instruments and need skillful analyst. To overcome these instrumental problems, some simple analytical techniques for authentication of high edible oils have been proposed, developed and standardized. One of the ideal methods providing fast and reliable results is Fourier transform infrared (FTIR) spectroscopy due to its property as the fingerprint technique, especially in combination with multivariate analysis (chemometrics).

FTIR spectroscopy combined with some chemometrics techniques of multivariate calibration of partial least square and discriminant analysis have been successfully used for authentication of VCO from some lower-priced fats and oils, namely lard from palm kernel oil [13], VCO from palm oil [14], corn and sunflower oils [15]. FTIR spectroscopy in combination with chemometrics was also used for authentication of candlenut [16] and for analysis of fatty acids [17]. However, the application of FTIR spectroscopic method for authentication of soybean oil and grape seed oil having a different characteristic with those oils has not been reported yet. Therefore, the objective of this research was to develop FTIR spectroscopy in combination with chemometrics of multivariate calibrations and discriminant analysis for quantification and classification of GSO and SO in VCO for authentication purposes.

\section{MATERIALS AND METHODS}

\section{Materials}

The used oils in this study, namely virgin coconut oil (VCO), grapeseed oil (GSO), and soybean oil (SO) with different brands were purchased from Serdang, Selangor, Malaysia. In order to assure the authenticity that the used oils were not blended (adulterated) with other oils, the composition of fatty acid (FA) in the oils were used to confirm its purity. The profiles of FA in the evaluated oils were compared with those specified in Codex Allimentarius [18]. The oils are considered as authentic if FA composition meets the FA specification stated in Codex Allimentarius. The solvents and reagents used during this study were of pro-analytical grade and purchased from E. Merck (Darmstadt, Germany).

\section{Determination of fatty acid composition}

The levels of fatty acids (FAs) in VCO, GSO and SO are determined by gas chromatography equipped with flame ionization detector (GCFID), as described in our previous paper [14]. Fatty acids are not volatile so that they must be derivatized into their methyl esters to 
obtain fatty acid methyl esters (FAMEs) which are volatile before GC-FID measurement. Briefly, $1 \mathrm{ml}$ oil sample was added with $4 \mathrm{ml}$ of sodium hydroxide $0.5 \mathrm{M}$ in methanol, heated for $20 \mathrm{~min}$ under nitrogen. The mixture was then added with $5 \mathrm{ml}$ of boron trifluoride15\%, prepared freshly in methanol. After $2 \mathrm{~min}, 5 \mathrm{ml}$ of heptane and $2 \mathrm{ml}$ of saturated $\mathrm{NaCl}$ were added, shaken vigorously. Heptane phase (supernatant) was separated and added with anhydrous sodium sulfate. Supernatant containing FAMEs were separated using capillary column RTX-5 $(0.25 \mathrm{~mm}$ internal diameter, $30 \mathrm{~m}$ length, and $0.2 \mu \mathrm{m}$ film thickness; Restex Corp., Bellefonte PA). The temperature was programmed as $50 \mathrm{oC}$ (hold for $1 \mathrm{~min})$, increased to $240 \mathrm{oC}(8 \mathrm{oC} / \mathrm{min})$, and finally held at $240 \mathrm{oC}$ for $5 \mathrm{~min}$. The temperatures of the detector and injector were $240 \mathrm{oC}$. The carrier gas $\mathrm{N} 2$ was set at velocity of $6.8 \mathrm{ml} / \mathrm{min}$. Identification of FA was carried out by comparing retention times of FAMEs in sample oils with those in a mixture of 37 FAME standards (FAMEs, C4 to C24) from Sigma Chemicals (St. Louis, MO, USA). While quantitative analysis of FA was performed using the normalization area (relative percentage). The analysis was done in three triplicates $(\mathrm{n}=3)$.

\section{Quantitative analysis of grapeseed oil and soybean in VCO}

Quantitative analysis of GSO and SO as adulterants in VCO was aided with multivariate calibrations of principle component regression (PCR) and partial least square (PLS) regression. A set of 30 VCO samples containing GSO and SO in the concentration ranges of 1.0$50.0 \%(\mathrm{v} / \mathrm{v})$ was prepared and called with calibration samples. For preparing validation samples, 25 independent samples containing the mixture of VCO-GSO and VCO-SO with different concentrations covered in the concentration range of calibration samples were also prepared. The mixture of samples was shaken vigorously to assure homogenous samples before analysis using FTIR spectrophotometer.

\section{Discriminant analysis}

Discriminant analysis (DA), a supervised pattern recognition technique commonly used for classification among samples, was used to classify VCO and VCO mixed with GSO and SO (as adulterants). A set of 20 pure VCO (non-adulterated model) and 20 sets of VCO mixed with GSO and SO in the concentration range of $1-50 \%$ of GSO and SO (adulterated model) were also prepared. VCO and VCO mixed with GSO-SO were subjected to FTIR spectra measurement and classified using DA with the aid of TQ Analyst software.

\section{Measurement of FTIR spectra}

The oil samples are scanned using FTIR spectrophotometer (Nicolet 6700 from Thermo Nicolet Corp., Madison, WI) facilitated with the detector of DTGS (deuterated triglycine sulphate) as a detector and connected to OMNIC operating system software (Version 7.0 Thermo Nicolet). The sampling compartment was Smart Attenuated Total Reflectance kit (Smart ARK, Thermo Electron Corp.) composed of zink selenide (ZnSe) crystal. FTIR spectra of samples were measured at 4000-650 cm-1, using 32 scans with a resolution of 4 $\mathrm{cm}-1$. These spectra were subtracted against air spectrum as the background. These spectra were recorded as absorbance mode at each data point in triplicate.

\section{Chemometrics and statistical analyses}

The software TQ AnalystTM version 6 from Thermo electron Corporation (Madison, WI, USA) included in FTIR spectrophotometer was used for data analysis which included the modelling of calibration and validation models based on multivariate calibration (PLS and PCR) and for classification using discriminant analysis (DA). The spectral regions where the variations were observed were selected and optimized during analysis (PLS, PCR and DA).

Table 1: Fatty acid composition in virgin coconut oil (VCO), grapeseed oil (GSO), and soybean oil (SO)

\begin{tabular}{llll}
\hline Fatty acids & Fatty acid composition & Grape seed oil & Soybean oil \\
\cline { 2 - 3 } & Virgin coconut oil & nd & $0.05 \pm 0.00(\mathrm{na})$ \\
C8:0 & $7.81 \pm 0.36(4.6-10.0)$ & $0.05 \pm 0.00(\mathrm{na})$ & $0.09 \pm 0.00(\mathrm{nd}-0.1)$ \\
C10:0 & $6.08 \pm 0.31(5.0-8.0)$ & $0.01 \pm 0.00(\mathrm{nd})$ & $0.09 \pm 0.00(\mathrm{nd}-0.2)$ \\
C12:0 & $47.01 \pm 0.67(45.1-53.2)$ & $0.01 \pm 0.00(\mathrm{nd}-0.3)$ & $10.99 \pm 0.08(8.0-13.5)$ \\
C14:0 & $18.45 \pm 0.47(16.8-21.0)$ & $7.87 \pm 0.04(5.5-11.0)$ & $0.10 \pm 0.00(\mathrm{nd}-0.2)$ \\
C16:0 & $8.99 \pm 0.37(7.5-10.2)$ & $0.03 \pm 0.00(\mathrm{nd}-1.2)$ & $4.81 \pm 0.16(2.0-5.4)$ \\
C16:1 & $0.02 \pm 0.00(\mathrm{nd})$ & $3.45 \pm 0.18(3.0-6.5)$ & $21.61 \pm 0.76(17-30)$ \\
C18:0 & $3.19 \pm 0.23(2.0-4.0)$ & $22.40 \pm 0.10(12.0-28.0)$ & $52.97 \pm 0.70(48.0-59.0)$ \\
C18:1 & $6.23 \pm 0.28(5.0-10.0)$ & $63.44 \pm 0.08(58.0-78.0)$ & $6.72 \pm 0.05(4.5-11.0)$ \\
C18:2 & $1.37 \pm 0.05(1.0-2.5)$ & $0.81 \pm 0.06(\mathrm{nd}-1.0)$ & $0.35 \pm 0.01(0.1-0.6)$ \\
C18:3 & $0.10 \pm 0.00(\mathrm{nd}-0.2)$ & $0.23 \pm 0.01(\mathrm{nd}-1.0)$ & $0.39 \pm 0.02(\mathrm{nd}-0.5)$ \\
C20:0 & $0.01 \pm 0.00(\mathrm{na})$ & $0.41 \pm 0.01(\mathrm{nd}-0.3)$ & \\
C20:1 & nd & & \\
\hline
\end{tabular}

Values in parentheses are taken from reference values in Codex Allimentarius (2011). nd = not detected; na = not available

\section{RESULTS AND DISCUSSION}

Table 1 listed the composition of fatty acid (FA) contained in VCO, grapeseed oil (GSO) and soybean oil (SO), as determined using gas chromatography with flame ionization detector, as recommended in several standard methods such as Codex Allimentarius and the American Oil Chemists' Society (AOCS). Quantitative analysis of FAs was carried out using the normalization area, i.e. peak area of specific fatty acids was divided by the total peak area of all FAs in VCO, GSO and SO. FA profiles of VCO, GSO and SO were in agreement in the ranges appear in the standard of Codex Alimentarius commission [18]. Therefore, it can be deduced that VCO, GSO and SO are not adulterated or mixed with other oils and are suitable for authentication study of VCO. The main FA composed VCO was lauric acid (C12:0), one of medium fatty acid, believed to be responsible in health-beneficial activities. The addition of SO and GSO, in turn, would reduce the levels of lauric acid. This reduction level could be used as an indicative that VCO has been mixed with other oils.

\section{Quantitative analysis}

Fig. 1 showed FTIR spectra of VCO, GSO and SO at mid-infrared region (4000-650 cm-1). Each bands/peaks and shoulders are characteristics for FTIR spectra of triglyceride (TG). This is not surprising because the main components composed of edible fats and oils are TG. There are some bands and shoulders difference between VCO and two other oils, mainly at wavenumbers of about $3007 \mathrm{~cm}-1$ and $1654 \mathrm{~cm}-1$. Bands at wavenumbers of 3007 and $1654 \mathrm{~cm}-1$ were absent in FTIR spectrum of VCO. These bands, corresponding to stretching vibration of unsaturation degree $(=\mathrm{CH}$ vinyl and $\mathrm{C}=\mathrm{C}$ ), were observed in FTIR spectra of GSO and SO. Based on fatty acid composition, GSO and SO contained much more unsaturated fatty acids than VCO, therefore it was not surprising if VCO did not reveal bands at 3007 and $1654 \mathrm{~cm}-1$. The difference was also observed at wavenumbers of 1120-1095 cm-1, corresponding to ether (C-0) vibration. VCO showed one peak at 1117 $\mathrm{cm}-1$, while GSO and SO revealed two peaks at 1117 and $1097 \mathrm{~cm}-1$, respectively. These differences were used as the basis for classification and quantification of GSO and SO in VCO. 


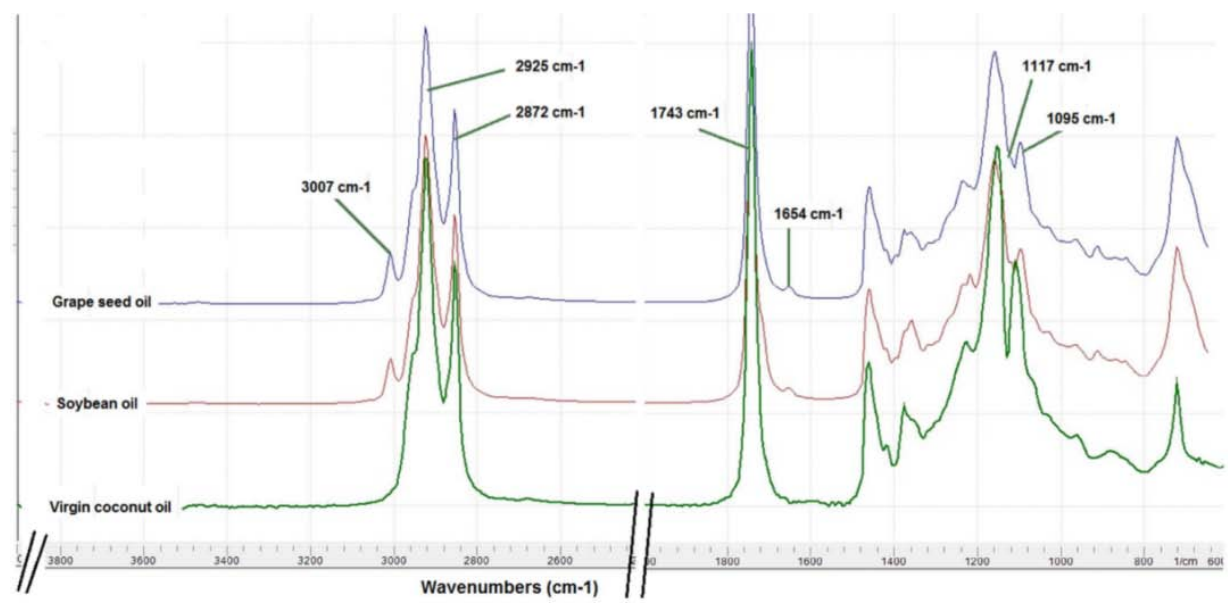

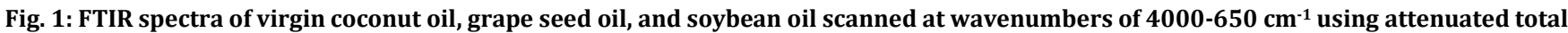
reflectance as the sampling technique

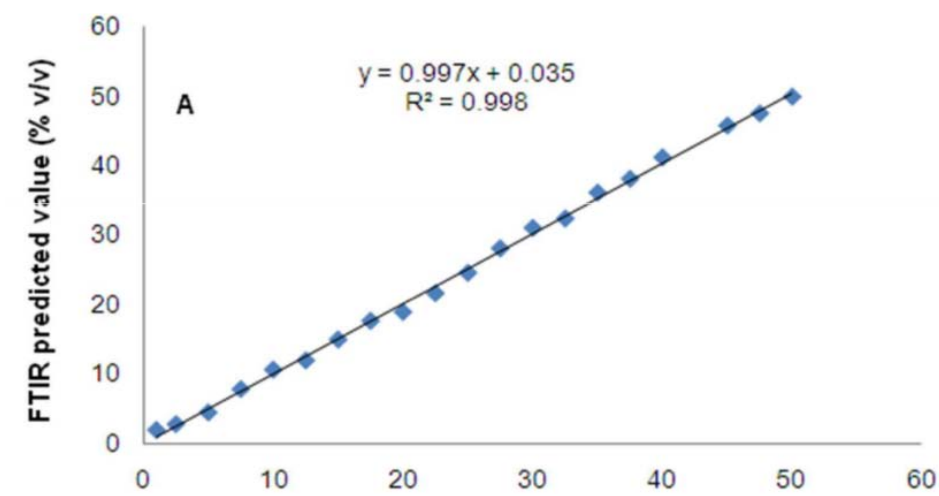

Actual value $(\%$ v/v)

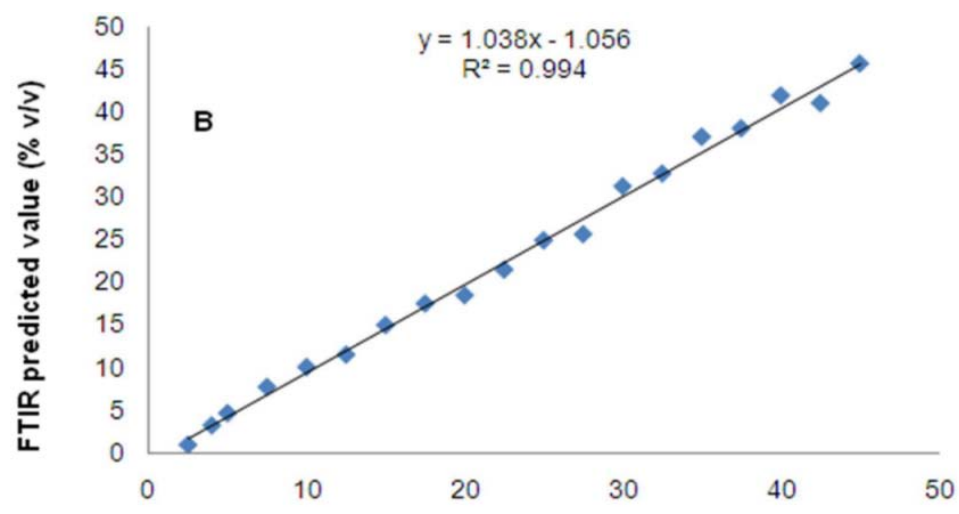

Actual value $(\%$ v/v $)$

Fig. 2: Linear regression for the relationship between actual value $(x)$ and FTIR predicted value $(y)$ of grape seed oil as adulterant in VCO. $A=$ calibration; $B=$ validation at combined frequency regions of $1200-9003027-2985 \mathrm{~cm}^{-1}$

In order to quantify the levels of GSO and SO as oil adulterants model in VCO, the performance of two multivariate calibrations of partial least square (PLS) and principal component regression (PCR) were compared. FTIR spectra were also treated with Savitzy-Golay derivatization (first and second derivatives) for making the comparison of analytical results obtained using normal and derivative spectra. Derivatization would improve the resolution of the overlapping peak, but it would decrease the sensitivity. The selection of multivariate calibration and spectra types were relied on some statistical parameters, namely coefficient of determination
$\left(\mathrm{R}^{2}\right)$, the number of factor, root mean square error of calibration (RMSEC), and root mean square error of prediction (RMSEP). The higher $\mathrm{R}^{2}$ and the lower number of factors, RMSEC and RMSEP were preferred for quantification of GSO and SO in VCO.

Table 2 compiled the performance of PLS and PCR for quantification modelling of GSO and SO in VCO. Based on table 2, FTIR at normal spectra at the combined wavenumbers of 1200-900 and 3027-2985 $\mathrm{cm}^{-1}$ assisted with PLS are chosen for quantification of GSO in VCO. PLS regression using FTIR normal spectra at combined 
wavenumbers of 1200-1000 and 3025-2995 $\mathrm{cm}^{-1}$ was suitable for quantification of GSO in VCO. Fig. 2 revealed the correlation between actual values of GSO (x-axis) and FTIR predicted values (y-axis), either in calibration or validation models. The $\mathrm{R}^{2}$ close to 1 indicated that calibration and validation models were accurate, while the low values of RMSEC and RMSEP indicated good precision. Similarly, the presence of SO in VCO was quantified using PLS calibration model using the combined spectral region of 1200-1000 and 3025-2995 $\mathrm{cm}^{-1}$. This wavenumbers region revealed spectral difference between SO and SO, there, it was very effective for making PLS model for the relationship between actual value and FTIR predicted values, as indicated by high $\mathrm{R}^{2}$ value and low RMSEC and RMSEP values.

Table 2: The performance of multivariate calibration of partial least square (PLS) and principle component regression (PCR) for quantitative analysis of grapeseed oil and soybean oil as adulterants in virgin coconut oil (VCO)

\begin{tabular}{|c|c|c|c|c|c|c|c|c|c|}
\hline \multirow{2}{*}{$\begin{array}{l}\text { Adulterants, } \\
\text { frequency } \\
\text { regions selected }\end{array}$} & \multirow{2}{*}{$\begin{array}{l}\text { Multivariate } \\
\text { calibration }\end{array}$} & \multirow[t]{2}{*}{ Spectra } & \multirow[t]{2}{*}{ Factor } & \multicolumn{2}{|l|}{ Equation } & \multicolumn{2}{|l|}{$\mathbf{R}^{2}$} & \multirow{2}{*}{$\begin{array}{l}\text { RMSEC } \\
(\%)\end{array}$} & \multirow{2}{*}{$\begin{array}{l}\text { RMSEP } \\
(\%)\end{array}$} \\
\hline & & & & Calibration & Validation & $\begin{array}{l}\text { Calib- } \\
\text { ration }\end{array}$ & $\begin{array}{l}\text { Predic } \\
\text { tion }\end{array}$ & & \\
\hline $\begin{array}{l}\text { Grape seed oil, } \\
1200-900\end{array}$ & PLS & Normal & 9 & $y=0.997 x+0.035$ & $y=1.038 x-1.056$ & 0.998 & 0.994 & 0.007 & 1.32 \\
\hline \multirow[t]{5}{*}{$3027-2985 \mathrm{~cm}-1$} & & 1 st der & 5 & $y=0.999 x+0.075$ & $y=0.907 x+2.040$ & 0.994 & 0.975 & 1.17 & 2.48 \\
\hline & & 2nd der & 10 & $y=0.966 x+0.433$ & $y=0.404 x+9.876$ & 0.991 & 0.409 & 0.091 & 11.9 \\
\hline & PCR & Normal & 10 & $y=0.993 x+0.098$ & $y=0.873 x+4.104$ & 0.998 & 0.981 & 0.622 & 2.80 \\
\hline & & 1st der & 10 & $y=0.997 x+0.091$ & $y=0.900 x+2.309$ & 0.994 & 0.973 & 1.19 & 2.61 \\
\hline & & 2nd der & 10 & $y=0.942 x+0.905$ & $y=0.259 x+14.66$ & 0.971 & 0.200 & 1.90 & 13.5 \\
\hline $\begin{array}{l}\text { Soybean oil, } \\
1200-1000\end{array}$ & PLS & Normal & 5 & $y=1.011 x-0.196$ & $y=1.042 x-1.098$ & 0.999 & 0.996 & 0.268 & 1.04 \\
\hline \multirow[t]{5}{*}{$3025-2995$} & & 1 st der & 5 & $y=0.999 x+0.011$ & $y=0.939 x+1.644$ & 0.999 & 0.989 & 0.334 & 1.70 \\
\hline & & 2nd der & 6 & $y=0.998 x+0.028$ & $y=0.762 x+3.380$ & 0.998 & 0.877 & 0.532 & 5.53 \\
\hline & PCR & Normal & 10 & $y=0.999 x+0.003$ & $y=0.990 x-0.187$ & 0.999 & 0.995 & 0.208 & 1.05 \\
\hline & & 1 st der & 10 & $y=0.999 x+0.010$ & $y=0.939 x+1.489$ & 0.999 & 0.990 & 0.337 & 1.64 \\
\hline & & 2nd der & 10 & $y=0.992 x+0.151$ & $y=0.780 x+3.759$ & 0.992 & 0.915 & 1.22 & 4.81 \\
\hline
\end{tabular}

*Spectral treatments and multivariate calibrations chosen for analysis of adulterants are italicized. PLS = partial least square; PCR = principle component regression; RMSEC = root mean square error of calibration; RMSEP = root mean square error of prediction.

\section{Discriminant analysis}

The chemometrics of discriminant analysis (DA), one of supervised pattern recognition techniques, was used for making classification between VCO and VCO adulterated with GSO and SO. The wavenumbers region used for quantitative analysis were used for classification. The Coomans plots for the fiestion of VCO adulterated with GSO and SO was shown in fig. 3. DA can classify pure VCO and that adulterated with GSO and SO with an accuracy level of $100 \%$.
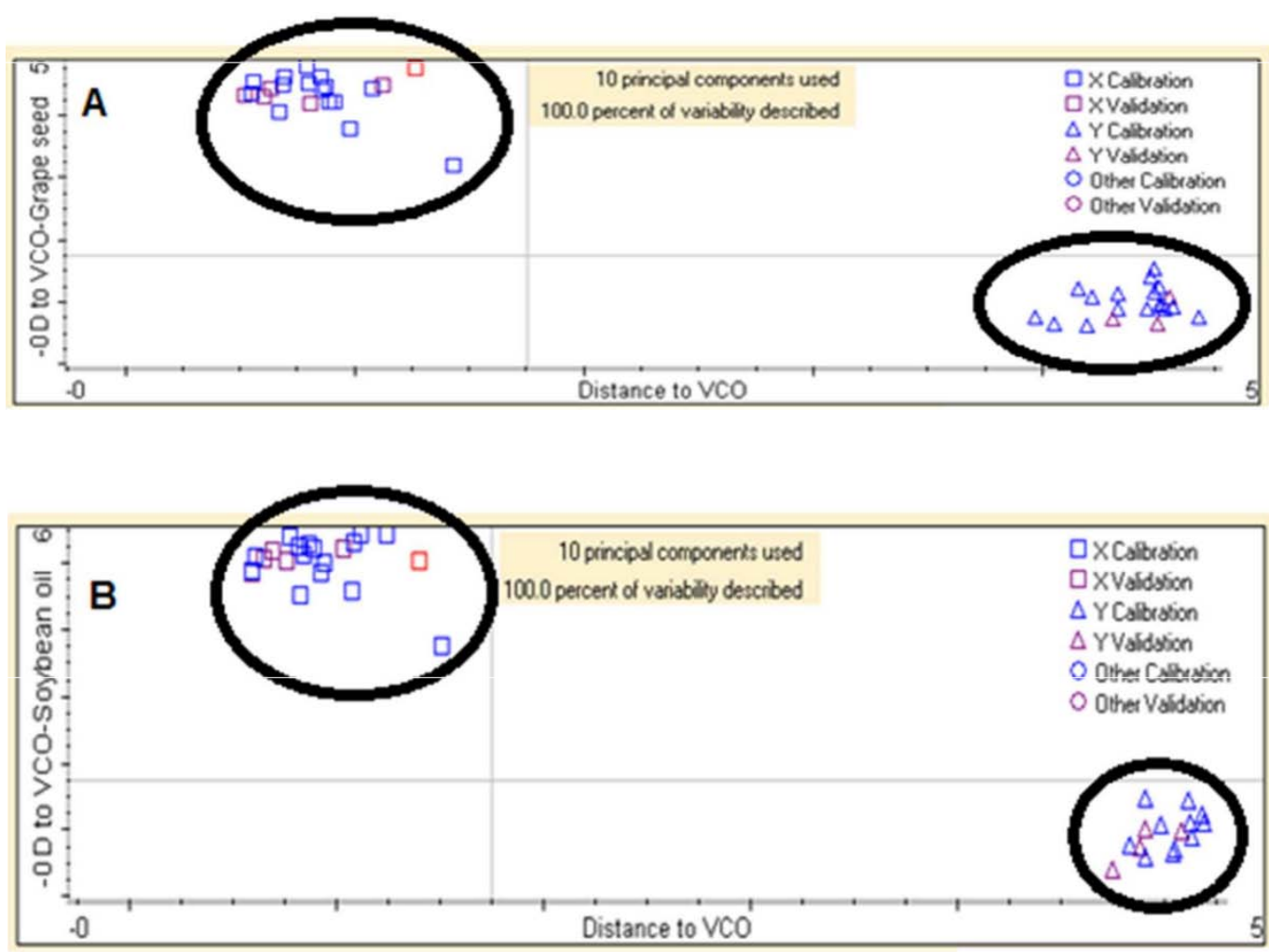

Fig. 3: The Coomans plot of virgin coconut oil (VCO) and adulterants: $(\square)$ V®; $(\Delta)$ VCO containing adulterants of canola oil (A); grapeseed oil (C); and soybean oil (F) 


\section{CONCLUSION}

FTIR spectroscopy combined with multivariate calibrations of PLS and PCR and discriminant analysis (DA) has been developed for adulteration analysis of VCO with GSO and SO. FTIR normal spectra combined with PLS is successfully used for quantification of GSO and SO with acceptable accuracy and precision. In addition, DA can classify VCO and VCO adulterated with GSO and SO accurately.

\section{ACKNOWLEDGMENT}

The first author thanks to the Ministry of Research and Higher Education for the financial support to the first author via program World Class University 2018 in Universiti Malaya.

\section{AUTHORS CONTRIBUTIONS}

AR performed research activities and prepared manuscript. YBCM and MEA designed research and made critical thinking on the manuscript.

\section{CONFLICTS OF INTERESTS}

All authors have none to declare

\section{REFERENCES}

1. Wang LL, Yang BK, Parkin KL, Jhonson EA. Inhibition of listeria monocytogenes by monoacylglycerols synthesized from coconut oil and milkfat by lipase-catalyzed glycerolysis. J Agric Food Chem 1993;41:1000-5.

2. Nevin KG, Rajamohan T. Influence of virgin coconut oil on blood coagulation factors, lipid levels and LDL oxidation in cholesterol-fed Sprague-dawley rats. E Spen Eur E J Clin Nutr Metab 2008;3:1-8.

3. Marina AM, Che Man YB, Amin I. Virgin coconut oil: emerging functional food oil. Trends Food Sci Technol 2009;20:481-7.

4. Craig Schmidt M, White MT, Teer P, Johnson J, Lane HW. Menhaden, coconut and corn oils and mammary tumor incidence in BALB/c virgin female mice treated with DMBA. Nutr Cancer 1993;20:99-106.

5. Mansor TST, Che Man YB, Shuhaimi M, Abdul Afiq MJ, Nurul FKM. Physicochemical properties of virgin coconut oil extracted from different processing methods. Int Food Res J 2012;19:837-45.

6. Alamprese C, Amigo JM, Casiraghi E, Engelsen SB. Identification and quantification of turkey meat adulteration in fresh, frozen- thawed and cooked minced beef by FT-NIR spectroscopy and chemometrics. Meat Sci 2016;121:175-81.

7. Basri KN, Hussain MN, Bakar J, Sharif Z, Abdul Khir MF, Zoolfakar AS. Classification and quantification of palm oil adulteration via portable NIR spectroscopy. Spectrochim Acta A Mol Biomol Spectrosc 2017;173:335-42.

8. Mansor TST, Che Man YB, Rohman A. Application of fast chromatography and fourier transform infrared spectroscopy for analysis of lard adulteration in virgin coconut oil. Food Anal Methods 2011;4:365-72.

9. Mansor TST, Che Man YB, Shuhaimi M. Employment of differential scanning calorimetry in detecting lard adulteration in virgin coconut oil. J Am Oil Chem Soc 2012;89:485-96.

10. Marina AM, Che Man YB, Amin I. Use of the SAW sensor electronic nose for detecting the adulteration of virgin coconut oil with RBD palm kernel olein. J Am Oil Chem Soc 2010;87:263-70.

11. Dayrit FM, Buenafe OEM, Chainani ET, de Vera IMS. Analysis of monoglycerides, diglycerides, sterols, and free fatty acids in coconut (Cocos nucifera L.) oil by $31^{\mathrm{P}}$ NMR spectroscopy. J Agric Food Chem 2008;56:5765-9.

12. Xu B, Li P, Ma F, Wang X, Matthäus B, Chen R, et al. Detection of virgin coconut oil adulteration with animal fats using quantitative cholesterol by GC x GC-TOF/MS analysis. Food Chem 2015;178:128-35.

13. Manaf MA, Che Man YB, Hamid NSA, Ismail A, Syahariza ZA. Analysis of adulteration of virgin coconut oil by palm kernel olein using Fourier transform Infrared spectroscopy. J Food Lipids 2007;14:111-21.

14. Rohman A, Che Man YB. Monitoring of virgin coconut oil (VCO) adulteration with palm oil using fourier transform infrared (FTIR) spectroscopy. J Food Lipids 2009;16:618-28.

15. Rohman A, Che Man YB. The use of fourier transform midinfrared (Ft-Mir) spectroscopy for detection and quantification of adulteration in virgin coconut oil. Food Chem 2011;129:583-8.

16. Yuliani F, Riyanto S, Rohman A. Application of FTIR spectra combined with chemometrics for analysis of candlenut oil adulteration. Int J Appl Pharm 2018;10:54-9.

17. Andina L, Saputri R, Putri AN, Rohman A. Infrared spectroscopy and multivariate calibration for the rapid quantification of free fatty acid content in pangasius hypopthalmus oil. Int J Appl Pharm 2018;10:199-203.

18. Codex Alimentarius Commission. Fats, Oils and Related Products, $2^{\text {Ed. }}$. Rome: Food and Agriculture Organization. The United Nations; 2001. 